\title{
Densidade de Carga Elétrica num Condutor Retilíneo Finito
}

\author{
(Electrical Charge density in finite rectilinear conductor) \\ Renê Robert \\ Departamento de Eletricidade da UFPR, CP 19047, Curitiba, Pr, 81531-990
}

Recebido em 15 de Outubro, 2003. Aceito em 28 de Novembro, 2003.

\begin{abstract}
Mostram-se dois métodos numéricos para o cálculo da distribuição de carga elétrica num condutor filiforme de comprimento finito e pequeno diâmetro. Os resultado numéricos são comparados com uma solução analítica aproximada mostrando boa concordância de resultados.

Two numerical methods for the calculation of electrical charge distribution on a finite linear wire of small diameter are shown. The obtained results are compared with an approximated analytical solution showing good results.
\end{abstract}

\section{Introdução}

Nos últimos anos tem-se discutido bastante o problema da distribuição de carga em um fio condutor retilíneo de comprimento finito, mantido a um potencial $\phi_{0}^{[1,2,3,4]}$, sendo que nenhum aborda o problema fazendo uso de técnicas numéricas. Do ponto de vista histórico este problema é bastante antigo ${ }^{[5]}$, pois foi resolvido analiticamente, de forma aproximada, em 1878 por J. C. Maxwell, sendo provavelmente um de seus últimos trabalhos de pesquisa em eletricidade e magnetismo ${ }^{[6]}$.

A maioria dos problemas de cálculo de campos elétricos e magnéticos apresentam soluções analíticas somente quando a geometria é extremamente simples. Nos problemas práticos esta geometria simples não existe e as dificuldades para obter soluções analíticas são insuperáveis. No passado, do ponto de vista técnico, este problema foi contornado em parte com o uso de técnicas experimentais ou gráficas. Atualmente lança-se mão de técnicas numéricas, pois essas são em geral as únicas possíveis ${ }^{[7]}$. Observa-se há algum tempo uma tendência em apresentar as técnicas numéricas para o cálculo de campos em livros de texto de eletromagnetismo o que é desejável para que os estudantes se tornem familiarizados com os diversos procedimentos.

Nosso objetivo é mostrar como obter a solução do problema da distribuição de cargas num fio retilíneo, finito e mantido a um potencial fixo utilizando duas técnicas numéricas simples: método de simulação de cargas e método dos momentos, e em seguida os resultados obtidos são comparados com aqueles calculados analiticamente. Estas técnicas podem ser usadas para resolver outros problemas simples, tais como a distribuição de carga em placas com formato retangular ou circular.

\section{Método de simulação de carga}

O princípio básico do método de simulação de carga (CSM) é muito simples ${ }^{[8]}$. Considere um condutor mantido a um potencial $\phi_{0}$ dentro do vácuo. A região de interesse é externa ao condutor. Sejam $n$ cargas pontuais $Q_{j},\{j=1,2,3, \ldots, n\}$ localizadas próximas à superfície do condutor, na região interna do mesmo e posicionadas pelo vetor $\vec{r}_{j}$. Este processo é em geral chamado de discretização do problema. O potencial elétrico num ponto $\mathrm{P}$ externo ao condutor posicionado pelo vetor $\vec{r}_{i}$, é dado pela equação

$$
\phi\left(\vec{r}_{i}\right)=\frac{1}{4 \pi \varepsilon_{0}} \sum_{j=1}^{n} \frac{Q_{j}}{\left|\vec{r}_{i}-\vec{r}_{j}\right|}=\sum_{j=1}^{n} p_{i j} Q_{j}
$$

em que $p_{i j}=\frac{1}{4 \pi \varepsilon_{0}} \frac{1}{\left|\vec{r}_{i}-\vec{r}_{j}\right|}$. Quando coloca-se o ponto definido pelo vetor $\vec{r}_{i}$ sobre $m$ pontos no contorno do condutor, a equação (1) pode ser escrita como

$$
\phi_{i}=\sum_{j=1}^{n} p_{i j} Q_{j} \text { com } i=1,2,3, \ldots, m .
$$

A equação (2) forma um sistema de $m$ equações lineares com $n$ incógnitas. Para nosso problema basta fazer $m=n \mathrm{e}$ o método é então chamado de método de simulação de carga convencional. A equação (2), quando escrita sob forma matricial, toma a forma

$$
\left[\phi_{i}\right]=\left[p_{i j}\right]\left[Q_{j}\right] .
$$

Em geral, com este método pode-se chegar a erros da ordem de $0,001 \%$ para valores grandes de $n$. Contudo, em pontas, cantos e eletrodos muito finos esta precisão dificilmente é alcançada.

Considere um condutor retilíneo de comprimento $2 c$ e raio $a$, sendo $a<<c$, mantido a um potencial constante $\phi_{0}$, conforme mostra a figura 1. Dividamos o comprimento $2 c$ sobre o eixo oz em $n-1$ partes iguais e coloquemos cargas pontuais $Q_{j}$ em cada um dos $n$ pontos. Analogamente dividamos a superfície do fio em $n-1$ partes iguais cujos pontos batiza-se pela letra $i$. O elemento de matriz $p_{i j}$ pode ser escrito como 


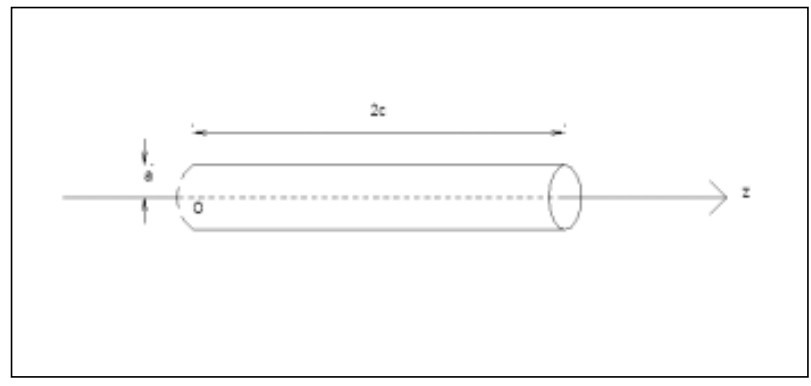

Figura 1. Cilindro condutor de raio $a$ e comprimento $2 c$ cujo eixo coincide com o eixo cartesiano $\mathrm{Oz}$.

$$
p_{i j}=\frac{1}{\sqrt{(i-j)^{2} \Delta^{2}+a^{2}}}
$$

em que $\Delta=\frac{2 c}{n-1}$ é a distância entre duas cargas consecutivas.

Como exemplo numérico façamos: $c=0,5 \mathrm{~m}, a=$ $0,001 \mathrm{~m}, \phi_{0}=1 \mathrm{~V}, 4 \pi \varepsilon_{0}=111,2626 \mathrm{pF} / \mathrm{m}$ e $n=1000$. Utilizando o software Mathematica ${ }^{\circledR}$, o cálculo numérico das cargas $Q_{j}$ fica simples e rápido. A figura 2 mostra o resultado obtido, em que a densidade linear de carga vale $\lambda_{j}=\frac{Q_{j}}{\Delta}$.

\section{Método dos momentos}

O método dos momentos (MM) é conceitualmente simples e muito usado em engenharia ${ }^{[9]}$. Para a finalidade deste trabalho vamos usar um caminho mais simples e adequado ao nível de graduação ${ }^{[10]}$. O potencial elétrico gerado por uma distribuição de cargas linear num ponto $\vec{r}$ é dado pela equação:

$$
\phi(\vec{r})=\frac{1}{4 \pi \varepsilon_{0}} \int_{\text {wire }} \frac{\lambda\left(z^{\prime}\right)}{\left|\vec{r}-\vec{r}^{\prime}\right|} d z^{\prime}
$$

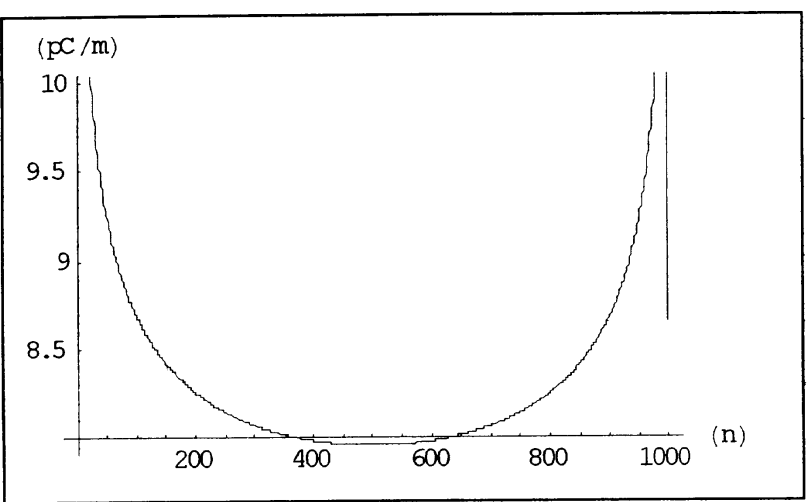

Figura 2. Distribuição linear de cargas no fio da figura 1 usando o método de simulação de cargas (CSM). Os parâmetros de cálculo usados foram: $c=0,5 \mathrm{~m}, c=0,001 \mathrm{~m} \phi_{0}=1 \mathrm{~V}, n=1000$. As linhas verticais que aparecem em torno de $n=0$ e $n=1000$ são aqui mostradas com o intuito de chamar atenção do leitor para a não confiabilidade dos resultados nestes pontos.

em que $\lambda\left(z^{\prime}\right)$ é a densidade de carga linear sobre o fio da figura 1, localizada pelo vetor $\vec{r}^{\prime}$. Quando conhecemos o potencial $\phi(\vec{r})$ e desconhecemos $\lambda\left(z^{\prime}\right)$ a equação (5) constitui uma equação integral de Fredholm de $1^{a}$. espécie. Para resolver numericamente esta equação integral, dividamos o fio em $n-1$ partes de comprimento $\Delta=\frac{2 c}{n-1}$ e chamemos de $\lambda_{j}$ a densidade linear de carga de cada parte. A equação (5) pode ser escrita para pontos sobre a superfície do fio como:

$$
\phi(\vec{r})=\frac{1}{4 \pi \varepsilon_{0}} \sum_{j=1}^{n} \lambda_{j} \int_{z_{j}}^{z_{j+1}} \frac{d z^{\prime}}{\sqrt{a^{2}+\left(z-z^{\prime}\right)^{2}}}
$$

Dividindo a superfície do fio em $n$ partes tal que $z=$ $\frac{\Delta}{2}+(i-1) \Delta \quad i=\{1,2,3 \ldots, n\}$ a equação (6) pode ser escrita como:

$$
4 \pi \varepsilon_{0} \phi_{0}=\sum_{j=1}^{n} \lambda_{j} \log \left|\frac{2(i-j)+1+\sqrt{\delta^{2}+(2(i-j)+1)^{2}}}{2(i-j)-1+\sqrt{\delta^{2}+(2(i-j)-1)^{2}}}\right|
$$

em que $\delta=\frac{2 a}{\Delta}$

Fazendo como antes, $c=0,5 \mathrm{~m}, a=0,001 \mathrm{~m}, \phi_{0}=1 \mathrm{~V}$, $\Delta \cong 0,001 \mathrm{~m}, 4 \pi \varepsilon_{0}=111,2626 \mathrm{pF} / \mathrm{m}, n=1000$ e usando o Mathematica ${ }^{\circledR}$ para o cálculo da equação (7), isto é, do sistema de $n$ equações lineares com $n$ incógnitas obtém-se o resultado espelhado na figura 3.

\section{Comparação entre os resultados numéricos e analítico}

Em artigo recente, J.D. Jackson ${ }^{[4,5]}$ deduziu uma equação aproximada que permite calcular a densidade de carga em um fio retilíneo de comprimento $2 c$ e secção transversal circular de raio $a$. Esta equação até a ordem de $O\left(\frac{1}{\Lambda^{3}}\right)$ é dada por

$$
\lambda(z)=\lambda_{0}\left\{1-\frac{1}{\Lambda} \ln \left(1-\xi^{2}\right)+\frac{1}{\Lambda^{2}}\left[\left(\ln \left(1-\xi^{2}\right)^{2}+\frac{1}{2}\left(\ln \left(\frac{1+\xi}{1-\xi}\right)\right)^{2}-\frac{\pi^{2}}{6}\right]\right\}\right.
$$




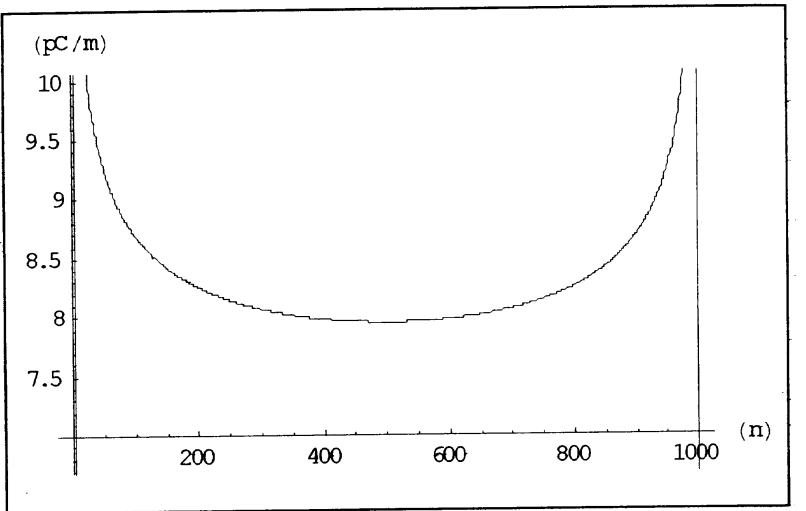

Figura 3. Distribuição linear de cargas no fio da figura 1 usando o método dos momentos (MM). Os parâmetros de cálculo usados foram: $c=0,5 \mathrm{~m}, a=0,001 \mathrm{~m}, \phi_{0}=1 \mathrm{~V}, n=1000$. As linhas verticais que aparecem em torno de $n=0$ e $n=1000$ são aqui mostradas com o intuito de chamar atenção do leitor para a não confiabilidade dos resultados nestes pontos.

em que $\Lambda=2 \ln \left(\frac{2 c}{a}\right), \xi=\frac{z}{c}$ e $\lambda_{0}=\frac{4 \pi \varepsilon_{0} \phi_{0}}{\Lambda}$. Quando o fio for mantido a um potencial $\phi_{0}=1 \mathrm{~V}, \lambda_{0}=8,0533 \mathrm{pC} / \mathrm{m}$ para o fio acima especificado.

Na figura 4 mostram-se os resultados obtidos com os métodos CSM, MM e a expressão analítica fornecido pela equação (8). Os cálculos são executados no intervalo do comprimento do condutor $[-0,45 \mathrm{~m}, 0,45 \mathrm{~m}]$. A maior diferença entre os resultados numéricos é de $0,2 \%$ e entre os resultados analítico e numéricos é de $0,4 \%$. A figura 5 mostra que a diminuição do raio $a$ do fio diminui o valor da densidade de carga e esta torna-se mais uniforme ${ }^{[1,4]}$, sendo os resultados numéricos e analíticos compatíveis entre si. É importante frisar que os cálculos numéricos não fornecem bons resultados nos extremos do fio.

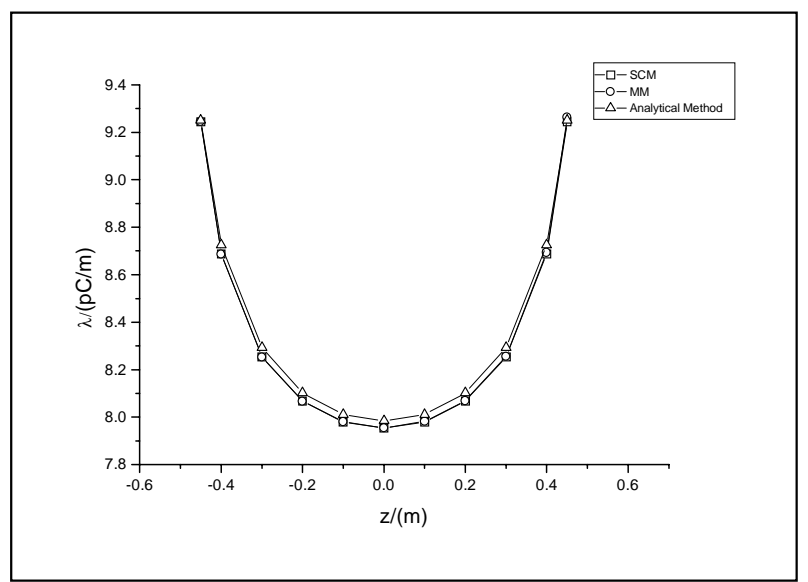

Figura 4. Comparação entre os métodos numéricos CSM, MM e analítico, usando como parâmetros $c=0,5 \mathrm{~m}, a=0,001 \mathrm{~m}$, $\phi_{0}=1 \mathrm{~V}$ e $n=1000 \quad z \in[-0,45-0,45] \mathrm{m}$.

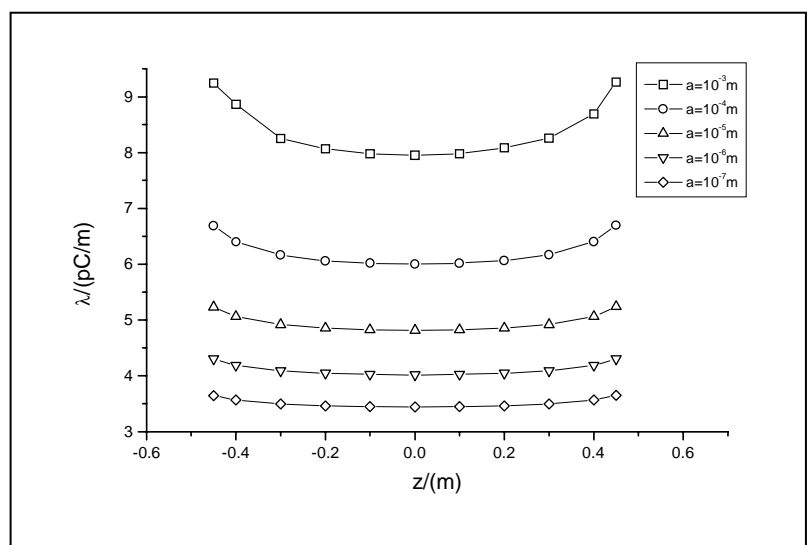

Figura 5. Comportamento da densidade linear de cargas quando a relação $a / c \rightarrow 0$, resultado este compatível com o de outros autores ${ }^{[1,4]}$. Os cálculos foram efetuados para $z \in$ $[-0,495-0,495] \mathrm{m}$ usando-se o método MM.

Uma informação adicional a se obter do calculo numérico é a capacitância do fio. Usando o método SCM obtém-se para a capacitância do fio acima especificado o valor. $C=8,499 \mathrm{pF}$, quando $n=1000$. A fórmula do artigo de J.D. Jackson ${ }^{[4]}$ fornece o valor, $C=8,457 \mathrm{pF}$ correspondendo portanto a uma diferença de $0,5 \%$. O leitor interessado pode consultar outras referências ${ }^{[11-14]}$ para o cálculo da distribuição de cargas em um fio mantido a potencial constante.

\section{Conclusão}

Uma grande quantidade de problemas práticos em eletrostática e na aproximação quase-estática, não envolvem a determinação de potenciais de uma dada distribuição de cargas, mas requerem que seja encontrado a distribuição de cargas que corresponde a alguns conjuntos de potenciais dados. O primeiro problema não é de todo fácil, pois, mesmo nos casos em que a distribuição das cargas pode ser descrita com simplicidade razoável, a avaliação da integral para o cálculo do campo pode ser difícil. Por outro lado, o problema inverso é de solução muito mais difícil, e soluções analíticas de fato somente existem para um número limitado de casos. Muitos problemas reais só podem ser tratados com sucesso por técnicas numéricas.

\section{Referências}

[1] D. J. GRIFFITHS and Y. LI, Charge Density on a Conducting Needle, Am. J. Phys. 64(6), June 1996. p. 706-714.

[2] R. H. GOOD, Comment on "Charge Density on a Conducting Needle", Am. J. Phys. 65(2), February 1997, p.155-156.

[3] M. ANDREWS, Equilibrium Charge Density on Conducting Needle, Am. J. Phys. 65(9), September 1997, p.846-850.

[4] J. D. JACKSON, Charge Density on Thin Straight Wire, Revisited, Am. J. Phys. 68(9), September 2000, p. 789-799.

[5] J. D. JACKSON, Charge Density on a Thin Straight wire: The First Visit, Am. J. Phys. 70(4), April 2002, p.409-410. 
[6] J. C. MAXWELL, The Scientific Papers of James Clerk Maxwell, Edited by W. D. Nivens, 2 vols. Bound as one, Dover Pub. Inc., N.Y. p.672-680.

[7] K. J. BINNS, P. J. LAWRENSON and C. W. TROBRIDGE, The Analytical and Numerical Solution of Electric and Magnetic Field, John Willey \& Sons, Baffins Lane, 1992.

[8] N. H. MALIK, A Review of the Charge Simulation Method and its Application, IEEE Transactions on Electric Insulation, vol.24 n.1 February 1989, p.3-20.

[9] R. HARRINGTON, Matrix Methods for Field Problems, Proceedings of the IEEE, vol.55, n.2, February 1967, p.136-149.

[10] L. L. TSAI and C.E SMITH, Moment Methods in Electro- magnetics for Undergraduates, IEEE Transactions on Education, vol.21, n.1, February 1978, p.14-22.

[11] P. SILVESTER, Modern Electromagnetic Field, PrenticeHall Inc., Englewood Cliffs, N.J., 1968, p.90-93.

[12] J. R REITZ, F. J. MILFORD and R.W. CHRISTY, Foundations of Electromagnetic Theory, Addison Wesley Publishing Co., Reading Massachusetts, 1993, p.84-89.

[13] N. N. O. SADIKU, Electromagnetics, Oxford University Press, N.Y., 1995, p.721-733.

[14] S. RATNAJEEVAN, H. HOOLE, P. RATNAMAHILAN and P. HOOLE, Engineering Electromagnetics, Oxford University Press, N.Y., 1996, p.176-179. 